$11 ; 10.2$

\title{
Синтез „нулей“ в диаграмме направленности частотно-независимой антенной решетки
}

\author{
(C) A.A. Ерохин \\ Сибирский федеральный университет, Красноярск, Россия \\ E-mail: aerokhin@sfu-kras.ru
}

Поступило в Редакцию 16 июля 2020г.

В окончательной редакции 29 сентября 2020 г.

Принято к публикации 25 декабря 2020г.

\begin{abstract}
Предложен метод синтеза „нулей“ в диаграмме направленности частотно-независимой антенной решетки. Проведены численные эксперименты для определения характеристик предложенного метода, выполнено сравнение с существующим методом синтеза в пространстве лучей.
\end{abstract}

Ключевые слова: антенная решетка, помехи, цифровое диаграммоформирование, диаграмма направленности.

DOI: 10.21883/PJTF.2021.07.50797.18469

Широкополосные антенные решетки (AР) с цифровым формированием диаграммы направленности (ДН) получают все большее распространение благодаря широкому спектру их применения в различных системах - от акустических [1] до телекоммуникационных [2]. В задачах широкополосного цифрового диаграммоформирования можно выделить класс АР с частотно-независимой ДН в широкой полосе частот [3]. Под частотно-независимой будем понимать АР, ДН которой не изменяется в полосе частот с коэффициентом перекрытия диапазона более двух. Использование цифровых фильтров с конечной импульсной характеристикой позволяет достичь широкополосных свойств диаграммы направленности AP [3]. Фильтры с конечной импульсной характеристикой (КИХ-фильтры) выполняют временну́ю фильтрацию, тем самым формируя частотно-зависимую характеристику для сигналов, принимаемых широкополосными антенными элементами.

Управление положением „нулей“ (провалов) в диаграмме направленности АР позволяет увеличить отношение сигнал/шум на выходе АР. Существующие методы синтеза „нулей“ в ДН частотно-независимых AP основаны, например, на разложении в пространстве лучей $[4,5]$ или на применении выпуклого программирования [6]. Метод в пространстве лучей требует для вычисления коэффициентов КИХ-фильтров выполнения преобразования Фурье и выбора маски частотно-независимой ДН, а для синтеза провалов необходимо решение задачи линейного программирования. Использование выпуклого программирования является вычислительно сложной задачей, особенно при большом количестве элементов АР и коэффициентов КИХ-фильтров.

В настоящей работе предложен метод синтеза „нулей“ в ДН частотно-независимой АР, основанный на свойствах ДН адаптивной АР. Для реализации предложенного метода требуется вычисление коэффициентов КИХ-фильтров по простым формулам и решение за- дачи линейного программирования (решение системы линейных уравнений с количеством переменных, равным количеству „нулей“ $)$.

Как известно, ДН узкополосной линейной адаптивной АР можно представить в виде суммы двух слагаемых - исходной (неискаженной) ДН и компенсационной ДН [7]:

$$
\dot{F}_{\Sigma}\left(\theta, \theta_{0}\right)=\dot{F}\left(\theta, \theta_{0}\right)-\dot{a} \dot{F}\left(\theta, \theta_{p}\right)
$$

где $\dot{F}\left(\theta, \theta_{i}\right)$ - диаграмма направленности АР, имеющая максимум в направлении угла $\theta_{i} ; \theta_{0}-$ направление на источник полезного сигнала; $\theta_{p}-$ направление на источник помехи; $\dot{a}-$ коэффициент нормировки. Этот вывод был независимо получен и в других работах [8-10]. Таким образом, данное свойство может применяться в методах синтеза „нулей“ в диаграмме направленности АР.

Запишем ДН узкополосной линейной АР с $K$ „нулями“ в заданных направлениях в виде взвешенной суммы ДН этой же АР с максимумами главного лепестка в $K$ направлениях [11]:

$$
\dot{F}(\theta)=\sum_{i=0}^{K} \alpha_{i} \dot{F}_{i}\left(\theta, \theta_{i}\right)
$$

где $\dot{F}\left(\theta, \theta_{i}\right)$ - диаграмма направленности, имеющая максимумы в направлениях $\theta_{i}(i=0$ определяет направление максимума суммарной ДН, $1 \leqslant i \leqslant K-$ направление „нулей“ суммарной ДН), $\alpha_{i}$ - неопределенные коэффициенты. Коэффициент $\alpha_{0}$ можно принять равным единице. Остальные коэффициенты $\alpha_{j}(1 \leqslant j \leqslant K)$ находятся из условий

$$
\dot{F}\left(\theta_{j}\right)=0, \quad 1 \leqslant j \leqslant K,
$$



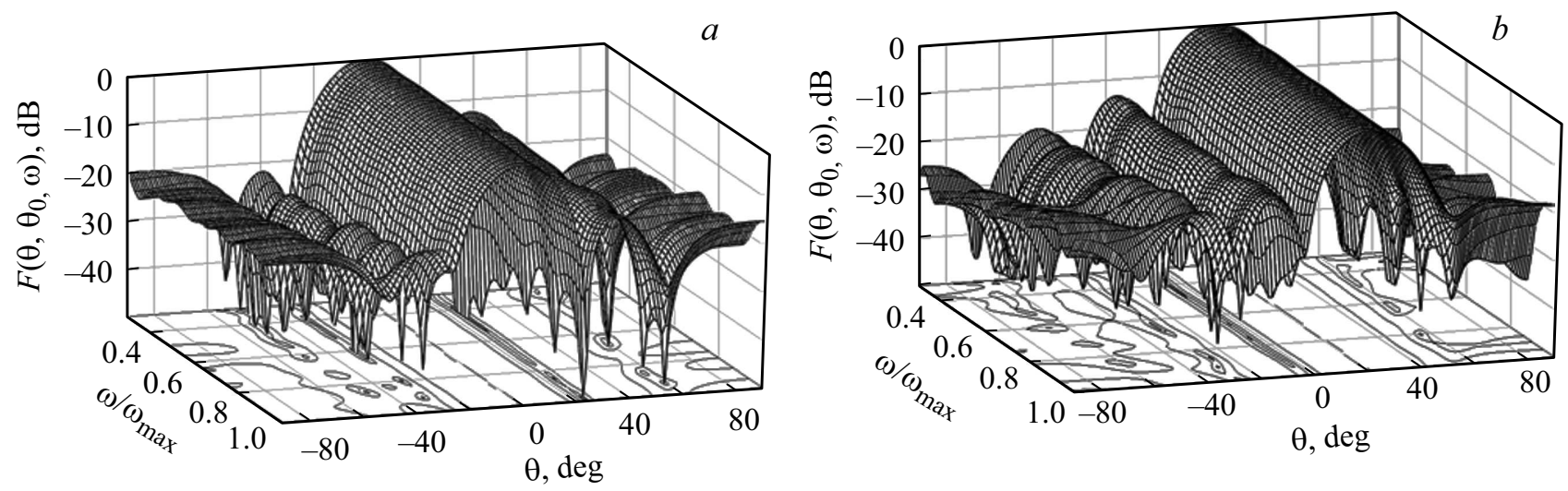

Рис. 1. Частотно-независимая ДН с синтезированными „нулями“, полученная прямым методом. $\theta_{0}=0(a)$ и $20^{\circ}(b)$.
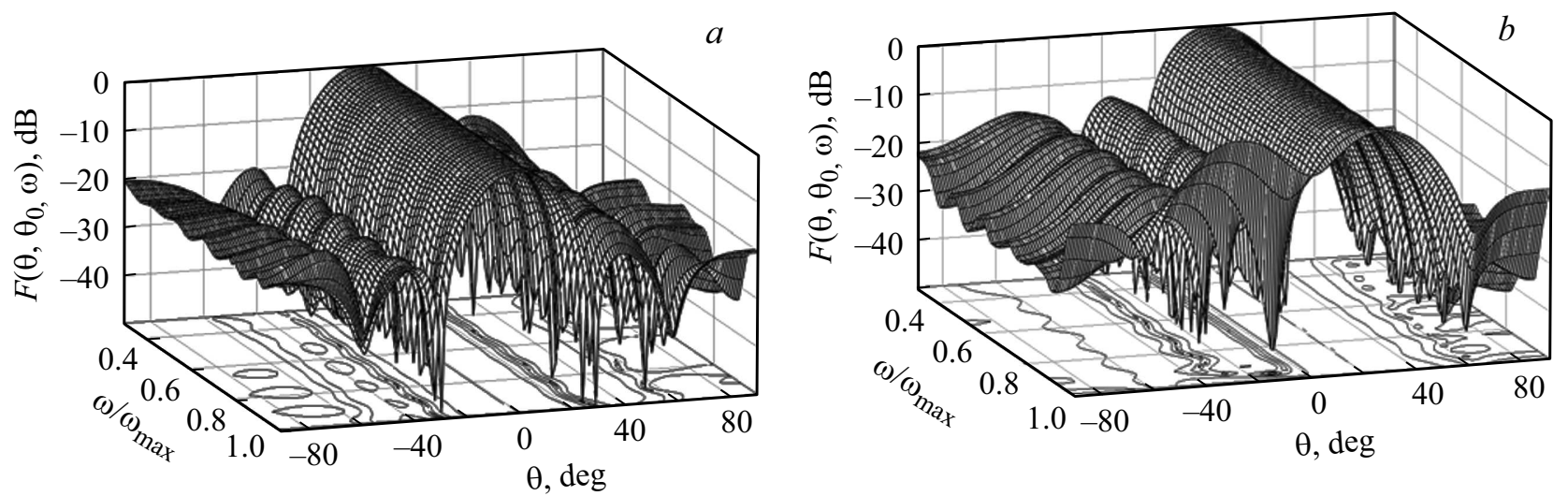

Рис. 2. Частотно-независимая ДН с синтезированными „нулями“, полученная методом BS-FFT. $\theta_{0}=0(a)$ и $20^{\circ}(b)$.

которые приведут к получению системы $K+1$ линейных однородных уравнений

$$
\left\{\begin{array}{l}
\sum_{i=0}^{K} \alpha_{i} \dot{F}_{i}\left(\theta_{0}, \theta_{i}\right)=1 \\
\sum_{i=0}^{K} \alpha_{i} \dot{F}_{i}\left(\theta_{1}, \theta_{i}\right)=0 \\
\vdots \\
\sum_{i=0}^{K} \alpha_{i} \dot{F}_{i}\left(\theta_{K}, \theta_{i}\right)=0
\end{array}\right.
$$

Далее будем рассматривать частотно-независимые линейные АР, причем ДН таких АР будем представлять в виде функции трех переменных $\dot{F}\left(\theta, \theta_{0}, \omega\right)$ : углов $\theta$, $\theta_{0}$ и частоты $\omega$. Для формирования „нуля“ частотнонезависимой ДН в заданном направлении необходимо решить систему уравнений (3). В левой части данной системы в качестве функции $\dot{F}\left(\theta_{j}, \theta_{i}\right)$ необходимо подставить частотно-независимую ДН на какой-либо фиксированной частоте $\dot{F}\left(\theta_{j}, \theta_{i}, \omega_{0}\right)$. Решив полученную систему уравнений, найдем коэффициенты $\alpha_{i}$. Для формирования ДН с частотно-независимыми провалами следует найти набор весовых коэффициентов (ВК), обеспечивающий реализацию необходимых частотных характеристик
КИХ-фильтров, входящих в состав широкополосной АР:

$$
\dot{h}_{n, m}^{\Sigma}=\sum_{i=0}^{K} \alpha_{i} h_{n, m}\left(\theta_{i}\right),
$$

где $h_{n, m}\left(\theta_{i}\right)$ - ВК КИХ-фильтров, формирующие такие частотные характеристики, чтобы отклонить максимум диаграммы направленности АР на угол $\theta_{i}$. Способы расчета ВК КИХ-фильтров можно найти в работах $[1,4-6,12,13]$ и др. ВК, полученные по выражению (4), будут обеспечивать формирование провалов в широкой полосе частот, но наименьшее значение ДН будет находиться на частоте, выбранной при составлении системы уравнений (3).

Будем кратко называть предложенный метод прямым. Предложенный способ синтеза „нулей“ в ДН частотно-независимой АР сравним с методом синтеза в пространстве лучей [4,5], который основан на использовании преобразования Фурье для создания ортогональных ДН, участвующих в формировании провалов в ДН (данный метод будем называть BS-FFT). Для этого будем формировать два „нуля“ ДН обоими методами в направлениях $\theta_{1}=60^{\circ}$ и $\theta_{2}=-30^{\circ}$ при одинаковом количестве антенных элементов в $\mathrm{AP}(N=16)$ и одинаковом количестве ВК КИХ-фильтров $(M=32)$. Элемен- 
Средние значения „нулей“ ДН (в dB)

\begin{tabular}{c|c|c|c|c|c}
\hline \multirow{2}{*}{ Метод } & \multicolumn{2}{|c|}{$\theta_{0}=0^{\circ}$} & \multicolumn{2}{c}{$\theta_{0}=20^{\circ}$} & \multirow{2}{*}{ Количество операций } \\
\cline { 2 - 5 } & $\theta_{1}=60^{\circ}$ & $\theta_{2}=-30^{\circ}$ & $\theta_{1}=60^{\circ}$ & $\theta_{2}=-30^{\circ}$ & \\
\hline Прямой & -30.4 & -34.4 & -33.4 & -31.7 & $2 N M(K+1)+(K+1)^{3}$ \\
BS-FFT & -33.0 & -33.5 & -31.9 & -33.5 & $4 L N_{1}^{2} \log _{2} N_{1}+L^{3}$
\end{tabular}

Пр имечан ие. $L-$ количество дополнительных лучей для метода BS-FFT $(L>K), N_{1}-$ размерность двумерного быстрого преобразования Фурье $\left(N_{1} \geqslant 3 J\right), J$ - количество элементов АР в маске ДН [4].

ты обеих АР расположены на расстоянии в половину длины волны на верхней частоте. При использовании прямого метода синтеза „нулей“ выберем нормированную частоту $\omega / \omega_{\max }=0.65$ для составления системы уравнений (3).

На рис. 1 и 2 показаны ДН с частотно-независимыми „нулями“, полученные различными методами при углах отклонения максимума ДН $\theta_{0}=0$ и $20^{\circ}$. Полученные ДН имеют частотно-независимые свойства в полосе нормированных частот $\omega / \omega_{\max } \approx[0.3 ; 1]$.

В таблице приведены средние значения провалов, синтезированных в ДН частотно-независимых АР двумя различными методами, и необходимое количество операций умножения и сложения для синтеза „нулей“ обоими методами. Из рис. 1, 2 и данных таблицы видно, что прямой метод и метод BS-FFT дают схожие результаты, при этом прямой метод не требует применения преобразования Фурье, выбора маски ДН, а также создания множества ортогональных ДН для синтеза „нулей“, т.е. обладает большей производительностью.

Таким образом, в работе метод синтеза „нулей“ в ДН распространен на случай частотно-независимых АР. Проведены численные эксперименты по синтезу „нулей“ в ДН частотно-независимых АР. Как показывают результаты проведенных численных экспериментов, предложенный метод и метод BS-FFT показывают примерно одинаковые характеристики, при этом предложенный прямой метод является более простым в реализации.

\section{Финансирование работы}

Исследование выполнено при финансовой поддержке Российского фонда фундаментальных исследований в рамках научного проекта № 19-37-90029.

\section{Конфликт интересов}

Автор заявляет, что у него нет конфликта интересов.

\section{Список литературы}

[1] G. Huang, J. Chen, J. Benesty, IEEE/ACM Trans. Audio Speech Lang. Process., 26 (12), 2305 (2018). DOI: 10.1109/TASLP.2018.2862826

[2] F. Sohrabi, W. Yu, IEEE J. Select. Areas Commun., 30 (7), 1432 (2017). DOI: 10.1109/JSAC.2017.2698958
[3] W. Liu, S. Weiss, Wideband beamforming: concepts and techniques (John Wiley \& Sons, Ltd, Chichester, 2010).

[4] T. Sekiguchi, Y. Karasawa, IEEE Trans. Signal Process., 48 (1), 277 (2000). DOI: 10.1109/78.815503

[5] W. Lui, R. Wu, R.J. Langley, IEEE Trans. Antennas Propag., 55 (12), 3413 (2007). DOI: 10.1109/TAP.2007.910322

[6] Y. Liu, J. Cheng, K.D. Xu, S. Yang, Q.H. Liu, Y.L. Gou, IEEE Trans. Antennas Propag., 66 (11), 5835 (2018). DOI: 10.1109/TAP.2018.2862361

[7] S.P. Applebaum, IEEE Trans. Antennas Propag., 24 (5), 585 (1976). DOI: 10.1109/TAP.1976.1141417

[8] H. Steyskal, IEEE Trans. Antennas Propag., 30 (2), 273 (1982). DOI: 10.1109/TAP.1982.1142765

[9] S. Chatterjee, S. Chatterjee, A. Majumdar, IEEE Antennas Wireless Propag. Lett., 16, 2521 (2017). DOI: 10.1109/LAWP.2017.2728813

[10] О.Г. Вендик, С.А. Калинин, Д.С. Козлов, ЖТФ, 83 (10), 117 (2013).

[11] A.A. Erokhin, Yu.P. Salomatov, V.S. Panko, M.I. Sugak, in Int. Siberian Conf. on control and communications (SIBCON) (IEEE, 2016), p. 7491854. DOI: $10.1109 /$ SIBCON.2016.7491854

[12] O. Rosen, I. Cohen, D. Malah, Signal Process., 130, 365 (2017). https://doi.org/10.1016/j.sigpro.2016.07.019

[13] А.А. Ерохин, Е.Р. Гафаров, Ю.П. Саломатов, Изв. вузов. Радиоэлектроника, 63 (10), 608 (2020). https://doi.org/10.20535/S0021347020100027 